\title{
Structure prediction and synthesis of a new class of macrocyclic peptide natural products
}

Graham A. Hudson ${ }^{\dagger}$, Annie R. Hooper ${ }^{\dagger}$, Adam J. DiCaprio ${ }^{\dagger \dagger}$, David Sarlah ${ }^{\dagger}$, Douglas A.

Mitchell ${ }^{\dagger *}$

$\dagger$ Department of Chemistry, University of Illinois at Urbana-Champaign, 600 South Mathews Avenue, Urbana, Illinois 61801, USA.

$\$$ Carl R. Woese Institute for Genomic Biology, University of Illinois at Urbana-Champaign, 1206 West Gregory Drive, Urbana, Illinois 61801, USA.

\section{ABSTRACT}

Owing to advances in genomic sequencing and bioinformatics, the breadth of natural product biosynthetic gene clusters (BGCs) has meteorically risen. This remains true for ribosomally synthesized and post-translationally modified peptides (RiPPs), where the rate of bioinformatically identifying clusters vastly outpaces characterization efforts. Uniting bioinformatics and enzymological knowledge to predict the chemical product(s) of a RiPP BGC with total chemical synthesis to obtain the natural compound is an effective platform for investigating cryptic gene clusters. Herein, we report the bioinformatic identification of a biosynthetically divergent class of RiPP bearing a subset of enzymes involved in thiopeptide biosynthesis. These natural products were predicted based on BGC architecture to undergo a formal, enzymatic [4+2]cycloaddition with subsequent elimination of the leader peptide and water to produce a tri-substituted pyridine-based macrocycle. Bearing a pyridine similar to thiopeptides but lacking the ubiquitous thiazole heterocycles, these new RiPPs were termed pyritides. One of the predicted natural products was chemically synthesized using an 11-step synthesis. This structure was verified to be chemically identical by an orthogonal chemoenzymatic synthesis utilizing the precursor peptide and the cognate [4+2]-cycloaddition enzyme. The chemoenzymatic platform was used to synthesize a second in-cluster pyritide product as well as analogs from other bioinformatically identified pyritide BGCs. This work exemplifies complementary bioinformatic, enzymological, and synthetic techniques to characterize a structurally distinct class of RiPP natural product.

\section{INTRODUCTION}

Advances in sequencing technology and analysis have rapidly expanded our knowledge of natural product biosynthetic space. ${ }^{1,2}$ This is notably true for the ribosomally synthesized and post-translationally modified peptide (RiPP) natural products, where characterization of new natural products is extensively outpaced by an ever-increasing amount of sequenced biosynthetic gene clusters (BGCs). ${ }^{3-6}$ Characterizing novel compounds often involves laborious and time-consuming techniques endemic to natural product discovery, such as the traditional "grind-and-find" approach or targeted heterologous expression of BGCs in tractable hosts. ${ }^{7}$ Given sufficient knowledge of the enzymes involved in biosynthetic maturation, the structure of a mature natural product can often be predicted, permitting access to the natural compound via 
chemical synthesis. ${ }^{8-11}$ This approach provides a new entry point in exploring natural product biosynthetic space. RiPPs are attractive targets for genome-guided total synthesis, as their structures are more readily predicted than other natural product classes given their direct ribosomal origin. ${ }^{12,13}$ Synthetic approaches also boast unparalleled capacity for generating analogs, often with significant scalability, obviating potential issues with isolation from producing organisms/hosts.

Recently, we reported a survey of the biosynthetic space for the thiopeptides, an extensively modified RiPP class. ${ }^{14}$ All thiopeptides feature azol(in)e heterocycles, dehydroamino acids, and a (dehydro)piperidine or pyridine heterocycle, but many contain a significant number of ancillary modifications beyond these three ubiquitous post-translational modifications (PTMs). ${ }^{12,14,15}$ The class-defining PTM for thiopeptides is the 6-membered nitrogenous heterocycle, which arises from a formal [4+2]-cycloaddition. We sought to determine if homologs of the thiopeptide thiomuracin [4+2]-cycloaddition enzyme, TbtD, ${ }^{15,16}$ occurred in any context beyond thiopeptide BGCs and performed a bioinformatic search for homologs that did not cooccur with the normal repertoire of thiopeptide enzymes. This search yielded several BGCs lacking a local azoline-forming cyclodehydratase while still possessing a split LanB, putatively responsible for Ser/Thr glutamylation and elimination to yield dehydroalanine (Dha) or dehydrobutyrine (Dhb), respectively, ${ }^{17,18}$ as well as a [4+2]-cycloaddition enzyme (Figures 1A and S2). ${ }^{19,20}$ The precursor peptides for these BGCs generally lacked Cys. Therefore, we reasoned that these BGCs would produce a structurally unique class of RiPP featuring a tri-substituted pyridine-based macrocycle and termed this still theoretical class as "pyritides."

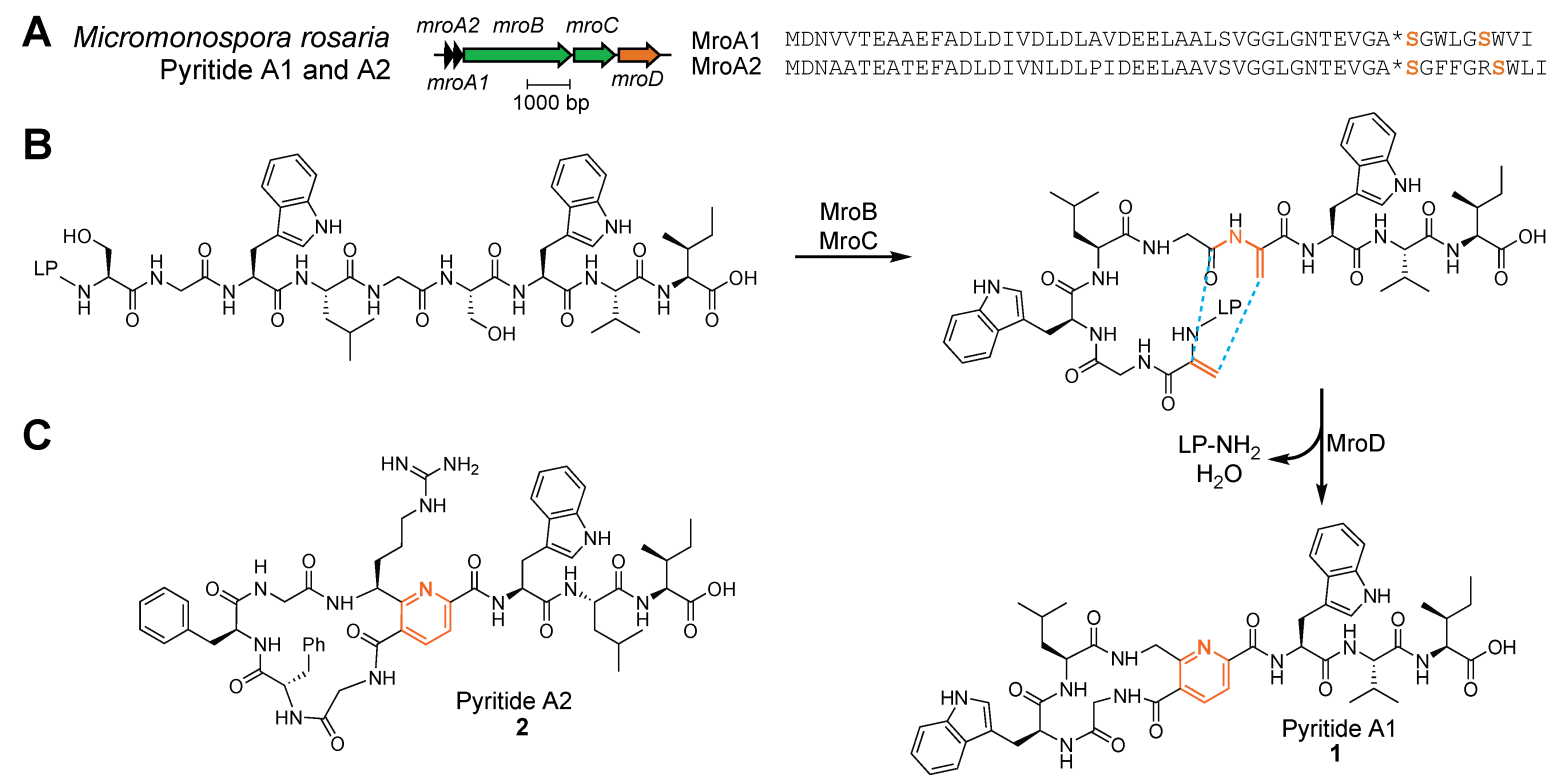

Figure 1. Biosynthesis of pyritides. (A) BGC from Micromonospora rosaria, which encodes a split LanB dehydratase and [4+2]-cycloaddition enzyme and notably lacks an azoline-forming cyclodehydratase. (B) Hypothetical biosynthetic route to produce pyritide A1 (1). (C) Predicted structure of pyritide A2 (2). LP, leader peptide.

We endeavored to test our structural hypothesis using a pyritide BGC identified in Micromonospora rosaria owing to strain availability and the fact that the two precursor peptides each contained only two Ser residues 
(Figure 1A). Thus, we felt confident in the structural prediction that both Ser would be converted to Dha prior to the formation of 14- and 17-atom macrocyclic pyritides by the [4+2]-cycloaddition enzyme, yielding pyritide A1 (1) and pyritide A2 (2), respectively (Figure 1B-C). Notably, 1 would contain a macrocycle 12atoms smaller than any characterized thiopeptide. As the predicted structure of the final natural products would be unambiguous, these structures would also be amenable to total chemical synthesis using the genome-guided structural prediction..$^{8-10}$

\section{RESULTS}

Using the predicted structure of 1 , a synthetic scheme consisting of 11 steps was devised (Figure 2A). To assess the validity of the structural prediction, we attempted to isolate 1 from the native producer; however, upon testing many different cultivation conditions, we were unsuccessful in eliciting production of the compound. Instead, building on our earlier work on thiomuracin biosynthesis, ${ }^{15,16}$ we opted to carry out a similar in vitro reconstitution of 1 using purified recombinant enzymes. In analogy to thiomuracin biosynthesis, we surmised Dha formation would require a tRNA-dependent glutamylation of Ser with subsequent elimination. In our hands, the enzymes responsible for this transformation have presented significant challenges for in vitro reconstitution. ${ }^{15,16}$ To obviate this issue and to leverage the fact that the precursor peptide of 1 (MroA1) lacked Cys, we hypothesized that replacement of Ser1 and Ser6 residues with Cys would enable a convenient desulfurization using dibromohexanediamide (3, DBHDA). ${ }^{20-23}$ The product of this reaction would be the putative substrate for an MroD-catalyzed [4+2]-cycloaddition (Figure 2B).

A
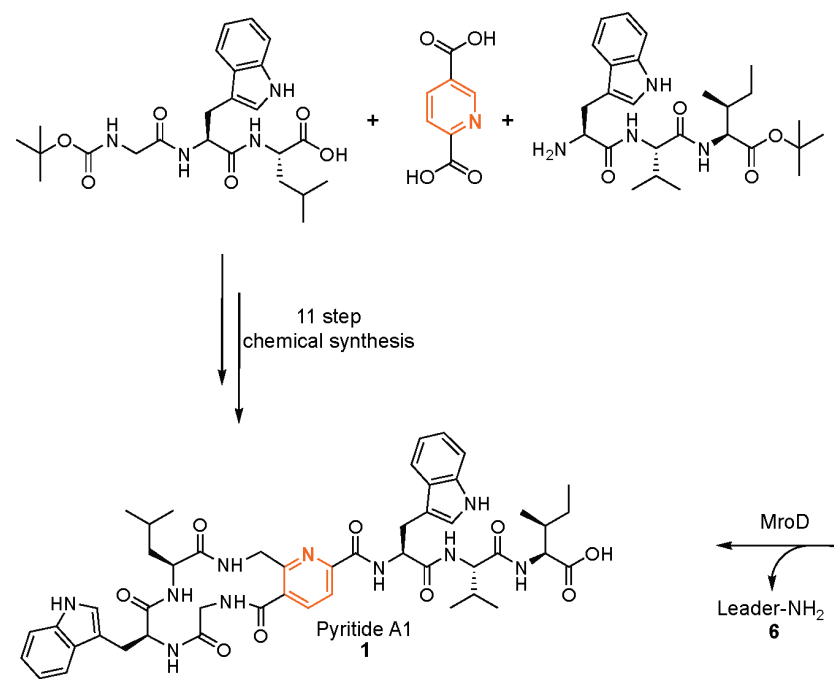

Figure 2. Orthogonal routes to obtain pyritide A1 (1). (A) Chemical synthesis beginning from pyridine-2,5-dicarboxylic acid and protected tripeptides. See Figure S3 for the enumerated route. (B) Chemoenzymatic synthesis using MroA1 precursor isolated from $E$. coli heterologous expression. The two Ser appearing in the wild-type sequence have been substituted with Cys to facilitate chemical desulfurization with DBHDA (3) and subsequent enzymatic [4+2]cycloaddition at the resultant Dha residues.
B

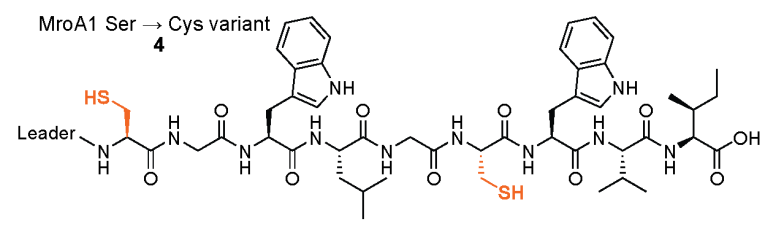

$\underbrace{\mathrm{H}_{2} \mathrm{~N}}_{\mathrm{Br}} \underbrace{\mathrm{NH}_{2}}_{\mathrm{O}} \underbrace{\mathrm{DBHDA}}_{\mathbf{3}}$

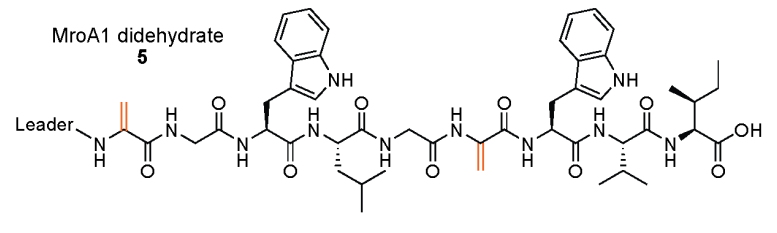


Pursuant to this, the maltose-binding protein (MBP)-tagged MroA1 precursor peptide featuring double substitution of Ser to Cys (4) as well as MroD were separately expressed in Escherichia coli and purified by affinity chromatography (Figure S1). After treatment with $\mathbf{3}$, we noted the formation of the doubly desulfurized species (5) by matrix-assisted laser desorption/ionization time-of-flight mass spectrometry (MALDI-TOF-MS, Figure 3). $\mathbf{5}$ was then presented to MroD, which resulted in the appearance of two new masses congruent with the predicted structure of 1 and the leader peptide with a C-terminal carboxamide, 6 (Figure 3).

1) MroA1 precursor peptide (S1C, S6C)

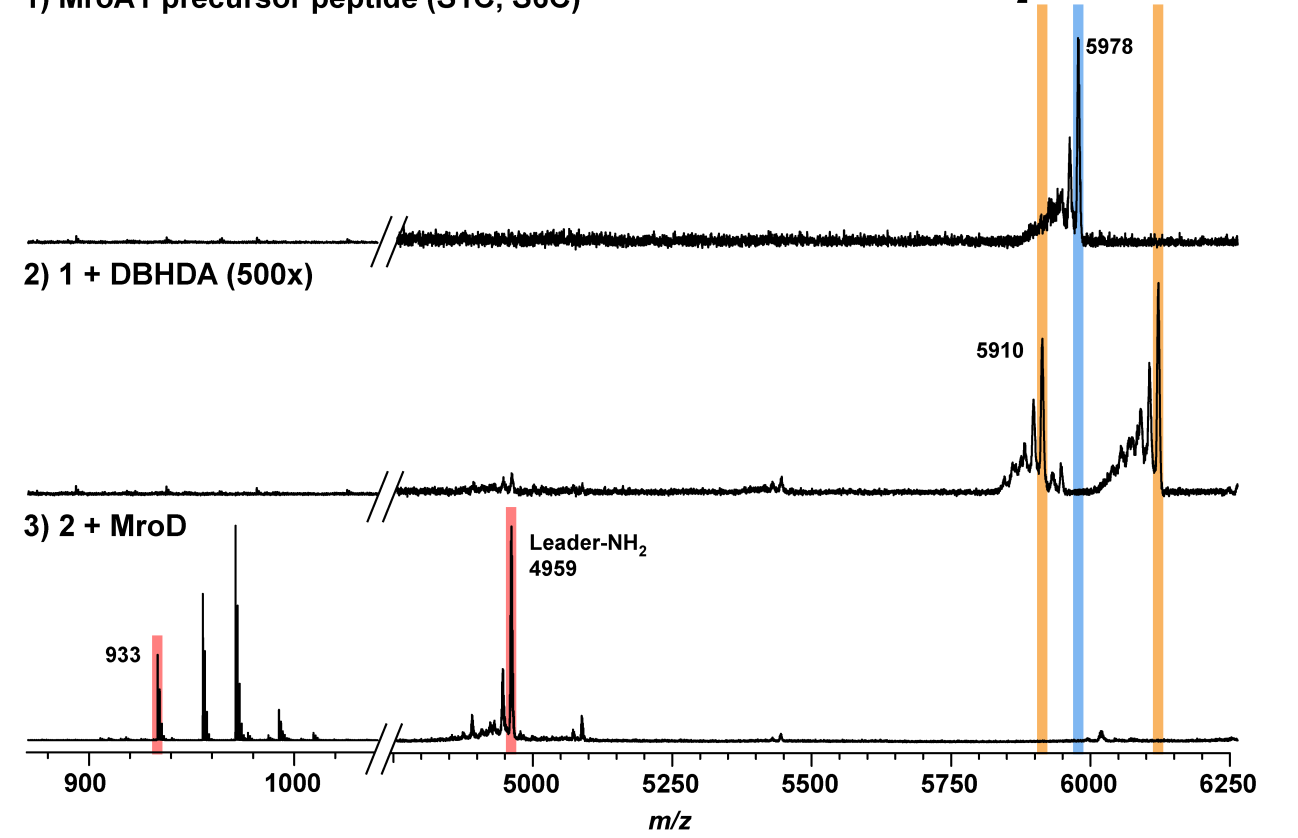

Figure 3. MALDI-TOF mass spectra detailing the chemoenzymatic synthesis of $\mathbf{1}$. The doubly Cys-substituted precursor peptide (4, m/z $5978 \mathrm{Da})$ was chemically desulfurized (5, m/z $5910 \mathrm{Da})$ and subsequently reacted with the [4+2]-cycloaddition enzyme MroD. The resulting masses correspond to the $[\mathrm{M}+\mathrm{H}]^{+}$ions predicted for $1(\mathrm{~m} / \mathrm{z} 933 \mathrm{Da})$ and the eliminated carboxamide leader peptide (6, $\mathrm{m} / \mathrm{z} 4959 \mathrm{Da})$.

The above chemoenzymatic route was then carried out on a larger scale to produce sufficient quantities of 1 to allow a detailed spectroscopic comparison to the synthetic standard. Approximately $20 \mathrm{~L}$ of MBP-MroA1 and $8 \mathrm{~L}$ of MBP-MroD were used to produce $\sim 2.5 \mathrm{mg}$ of 1 . Several analytical techniques were used to characterize the synthetic and chemoenzymatic samples of $\mathbf{1}$, including high-resolution and tandem mass spectrometry (HR-MS/MS), $\mathrm{C}_{18}$ analytical HPLC retention times, and ${ }^{1} \mathrm{H}$ NMR (Figures S4S8, S16). Data from all three methods confirmed that the structure of 1 was correctly predicted and the synthetic and chemoenzymatically prepared samples were chemically indistinguishable.

Using the chemoenzymatic route, we next sought to obtain a serviceable quantity of pyritide A2 (2, Figure 1C). The principle difference between $\mathbf{1}$ and $\mathbf{2}$ is the presence of an Arg in $\mathbf{2}$ adjacent to the pyridine. This position is conserved in several other bioinformatically predicted pyritides (Figure S3). Akin to 1, the MroA2 precursor was doubly substituted with Cys at both Ser positions, heterologously expressed, purified, 
and chemically desulfurized by 3 . This resulted in the production of a mass consistent with the predicted structure of 2 , which was verified by ${ }^{1} \mathrm{H}$ NMR and HR-MS/MS (Figures S9-S12), and the ejected carboxamide leader peptide $\mathbf{6}$. Analysis by HR-MS/MS confirmed that the leader peptide of MroA2 was eliminated as a C-terminal carboxamide (Figure S13). Purified 1 and 2 were then subjected to a brief panel of growth-suppression assays; however, we did not detect such activity for either pyritide tested alone or in combination (Table S2).

We next assessed the robustness of the chemoenzymatic route to determine if the MroD [4+2]cycloaddition enzyme could provide access to additional predicted pyritides without requiring the native producer. Thus, we prepared chimeric precursor peptides consisting of the leader region of MroA2 fused to the core region of other predicted precursors with the Ser-to-Cys replacement for positions that form the pyridine (Table S1). In the case Nonomuraea sp. WAC 01424, the precursor contained Cys, thus this residue was replaced with Ser to prevent over-desulfurization. These chimeric substrate peptides were heterologously expressed, purified, chemically desulfurized, and reacted with MroD. Upon MS analysis, we observed a pyridine-containing product for only two of the four chimeric substrates tested. The examples from Micromonospora yangpuensis and Nonomuraea sp. WAC 01424 were successful and produced analogs 7 and 8 respectively, while those from Herbidospora mongoliensis and Herbidospora yilanensis failed to generate product. For the former examples, HR-MS/MS was used to verify the composition of the product and the sites of macrocyclization (Figures S14-S15).

A

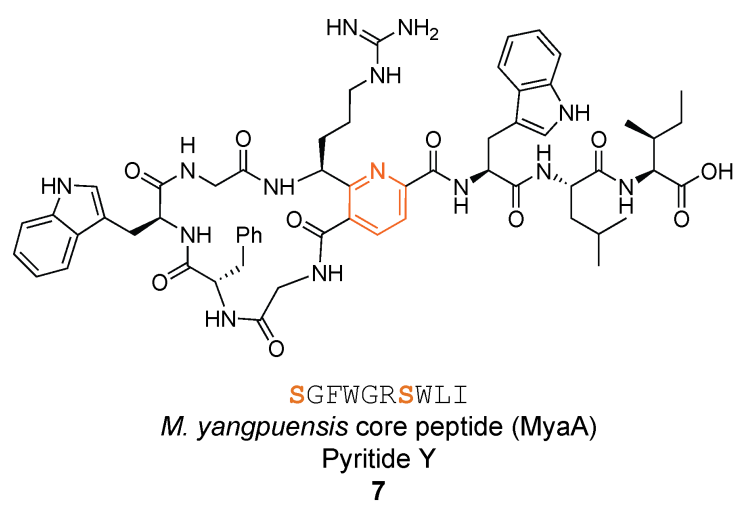

B

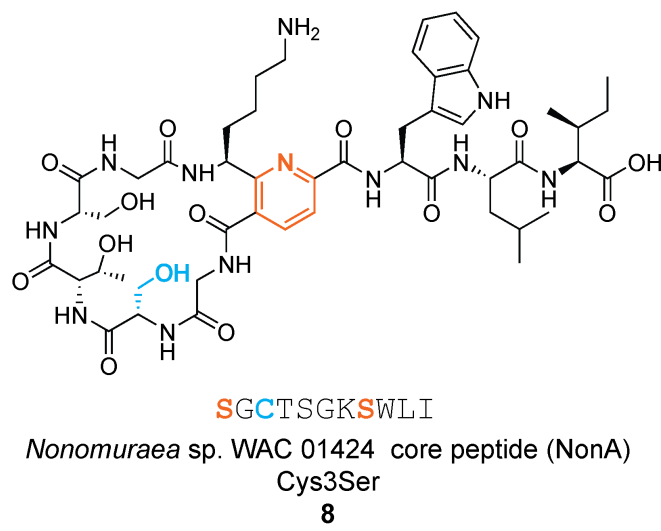

Figure 4. Pyritide compounds produced through chemoenzymatic synthesis utilizing chimeric precursor peptides. (A) Structure of pyritide $Y$ (7) from Micromonospora yangpuensis. Similar to $\mathbf{1}$ and 2, the structure of $\mathbf{7}$ can be unambiguously deduced as it only contains two pyridine-bound Ser (orange). (B) Structural analog of the 20-atom pyritide produced by Nonomuraea sp. WAC 01424 (8). This compound has numerous additional residues that may be modified (Ser, Thr, and Cys) and thus this structure will not be identical to the natural product. Cys 3 was substituted with Ser (blue) to prevent over-desulfurization.

Our data suggest that MroD displays some tolerance towards non-cognate substrates and is capable of producing pyridine-based macrocycles at least in the range of 14 to 20 atoms. Further studies will be required to fully elucidate the substrate tolerance of MroD and other pyritide [4+2]-cycloaddition enzymes. It should be noted that while the chemoenzymatic route offers convenient access to pyritide 
analogs, a drawback is the inability to install Dhb at Thr in ribosomally synthesized core peptides. The relative simplicity with which pyritide scaffolds can be accessed through total synthesis suggests an avenue to produce Dhb-containing scaffolds or analogs. The chemoenzymatic approach also relies on assuming the extent of dehydration to a precursor peptide. In the cases of 1, 2, and 7 , the resultant structures can be unambiguously deduced as only two Ser are present and both incorporated into pyridine. For other structures containing additional Ser or Thr residues, the natural structures are less obvious and require further exploration. In the unique case of the Nonomuraea precursor peptide, Cys is present and thus caution is warranted, as desulfurization chemistry may lead to an unnatural Dha.

\section{CONCLUSION}

The genomics revolution has led to a renaissance in natural product discovery. Massive growth in genome databases combined with the development of utilities to analyze and interpret these data has bolstered our knowledge of natural product biosynthetic space, most of which remains unexplored. Together, these advances have led to the discovery of numerous new natural products ${ }^{24-28}$ and, in the case of RiPPs, new post-translational modifications. ${ }^{14,29-35}$ Bioinformatics recently has been utilized to discover new RiPP classes, such as the $\alpha$-keto $\beta$-amino acid-containing peptides, ${ }^{36}$ RiPPs generating thiaglutamate by peptide-amino acyl tRNA ligases (PEARLs), ${ }^{33}$ aliphatic ether-containing rotapeptides ${ }^{31}$ and non- $\alpha$ thioether-containing ranthipeptides, ${ }^{37}$ as well as the pyritides, described herein. Bioinformatics offers a means to prioritize the characterization of divergent BGCs that give rise to novel compounds while also mitigating the historical issue of rediscovery. This paradigm shift is further enhanced by the unification of enzymology and synthetic chemistry as it provides a fruitful platform to access new molecules. Here, we have applied this methodology by identifying unusual context for homologs of thiopeptide [4+2]cycloaddition enzymes, leading to the structural prediction, chemical synthesis, and enzymatic verification of the founding members of a new RiPP class, the pyritides. We anticipate further investigations into pyritides will yield greater insights into the enzymology and biological function which will undoubtedly be augmented by complementary chemical synthesis. Moreover, we predict that the interplay between natural product enzymology and chemical synthesis will become an increasingly useful means producing new natural products or analogs thereof.

\section{AUTHOR INFORMATION}

\section{Corresponding Author}

* douglasm@illinois.edu

Author Contributions

The manuscript was written through contributions of all authors. All authors have given approval to the final version of the manuscript.

Funding Sources

This work was supported in part by grants from the National Institutes of Health (GM097142 to D.A.M.). 
Notes

The authors declare no competing financial interests.

\section{ACKNOWLEDGMENT}

We would like to thank Andraž Oštrek for assistance in synthetic scale up of 1.

\section{REFERENCES}

(1) Cimermancic, P.; Medema, M. H.; Claesen, J.; Kurita, K.; Wieland Brown, L. C.; Mavrommatis, K.; Pati, A.; Godfrey, P. A.; Koehrsen, M.; Clardy, J.; et al. Insights into Secondary Metabolism from a Global Analysis of Prokaryotic Biosynthetic Gene Clusters. Cell 2014, 158 (2), 412-421. https://doi.org/10.1016/j.cell.2014.06.034.

(2) Tietz, J. I.; Schwalen, C. J.; Patel, P. S.; Maxson, T.; Blair, P. M.; Tai, H.-C.; Zakai, U. I.; Mitchell, D. A. A New Genome-Mining Tool Redefines the Lasso Peptide Biosynthetic Landscape. Nat Chem Biol 2017, 13,470 . 4 https://doi.org/10.1038/nchembio.2319 https://www.nature.com/articles/nchembio.2319\#supplementary-information.

(3) de los Santos, E. L. C. NeuRiPP: Neural Network Identification of RiPP Precursor Peptides. Sci. Rep. 2019, 9 (1), 13406. https://doi.org/10.1038/s41598-019-49764-z.

(4) Blin, K.; Shaw, S.; Steinke, K.; Villebro, R.; Ziemert, N.; Lee, S. Y.; Medema, M. H.; Weber, T. AntiSMASH 5.0: Updates to the Secondary Metabolite Genome Mining Pipeline. Nucleic Acids Res. 2019, 47 (W1), W81-W87. https://doi.org/10.1093/nar/gkz310.

(5) van Heel, A. J.; de Jong, A.; Song, C.; Viel, J. H.; Kok, J.; Kuipers, O. P. BAGEL4: A User-Friendly Web Server to Thoroughly Mine RiPPs and Bacteriocins. Nucleic Acids Res. 2018, 46 (W1), W278-W281. https://doi.org/10.1093/nar/gky383.

(6) Skinnider, M. A.; Johnston, C. W.; Edgar, R. E.; Dejong, C. A.; Merwin, N. J.; Rees, P. N.; Magarvey, N. A. Genomic Charting of Ribosomally Synthesized Natural Product Chemical Space Facilitates Targeted Mining. Proc. Natl. Acad. Sci. 2016, 113 (42), E6343. https://doi.org/10.1073/pnas.1609014113.

(7) Bachmann, B. O.; Van Lanen, S. G.; Baltz, R. H. Microbial Genome Mining for Accelerated Natural Products Discovery: Is a Renaissance in the Making? J. Ind. Microbiol. Biotechnol. 2014, 41 (2), 175-184. https://doi.org/10.1007/s10295-013-1389-9.

(8) Vila-Farres, X.; Chu, J.; Ternei, M. A.; Lemetre, C.; Park, S.; Perlin, D. S.; Brady, S. F. An Optimized Synthetic-Bioinformatic Natural Product Antibiotic Sterilizes Multidrug-Resistant Acinetobacter BaumanniiInfected Wounds. mSphere 2018, 3 (1), e00528-17. https://doi.org/10.1128/mSphere.00528-17.

(9) Chu, J.; Vila-Farres, X.; Inoyama, D.; Ternei, M.; Cohen, L. J.; Gordon, E. A.; Reddy, B. V. B.; Charlop-Powers, Z.; Zebroski, H. A.; Gallardo-Macias, R.; et al. Discovery of MRSA Active Antibiotics Using Primary Sequence from the Human Microbiome. Nat. Chem. Biol. 2016, 12 (12), 1004-1006. https://doi.org/10.1038/nchembio.2207.

(10) Chu, J.; Vila-Farres, X.; Inoyama, D.; Gallardo-Macias, R.; Jaskowski, M.; Satish, S.; Freundlich, J. S.; Brady, S. F. Human Microbiome Inspired Antibiotics with Improved $\beta$-Lactam Synergy against MDR Staphylococcus Aureus. ACS Infect. Dis. 2018, 4 (1), 33-38. https://doi.org/10.1021/acsinfecdis.7b00056.

(11) Chu, J.; Vila-Farres, X.; Brady, S. F. Bioactive Synthetic-Bioinformatic Natural Product Cyclic Peptides Inspired by Nonribosomal Peptide Synthetase Gene Clusters from the Human Microbiome. J. Am. Chem. Soc. 2019, 141 (40), 15737-15741. https://doi.org/10.1021/jacs.9b07317. 
(12) Arnison, P. G.; Bibb, M. J.; Bierbaum, G.; Bowers, A. A.; Bugni, T. S.; Bulaj, G.; Camarero, J. A.; Campopiano, D. J.; Challis, G. L.; Clardy, J.; et al. Ribosomally Synthesized and Post-Translationally Modified Peptide Natural Products: Overview and Recommendations for a Universal Nomenclature. Nat Prod Rep 2013, 30 (1), 108-160. https://doi.org/10.1039/C2NP20085F.

(13) Funk, M. A.; van der Donk, W. A. Ribosomal Natural Products, Tailored To Fit. Acc. Chem. Res. 2017, 50 (7), 1577-1586. https://doi.org/10.1021/acs.accounts.7b00175.

(14) Schwalen, C. J.; Hudson, G. A.; Kille, B.; Mitchell, D. A. Bioinformatic Expansion and Discovery of Thiopeptide Antibiotics. J. Am. Chem. Soc. 2018, 140 (30), 9494-9501. https://doi.org/10.1021/jacs.8b03896.

(15) Hudson, G. A.; Zhang, Z.; Tietz, J. I.; Mitchell, D. A.; van der Donk, W. A. In Vitro Biosynthesis of the Core Scaffold of the Thiopeptide Thiomuracin. J Am Chem Soc 2015, 137 (51), 16012-16015. https://doi.org/10.1021/jacs.5b10194.

(16) Zhang, Z.; Hudson, G. A.; Mahanta, N.; Tietz, J. I.; van der Donk, W. A.; Mitchell, D. A. Biosynthetic Timing and Substrate Specificity for the Thiopeptide Thiomuracin. J Am Chem Soc 2016, 138 (48), 1551115514. https://doi.org/10.1021/jacs.6b08987.

(17) Bothwell, I. R.; Cogan, D. P.; Kim, T.; Reinhardt, C. J.; van der Donk, W. A.; Nair, S. K. Characterization of Glutamyl-TRNA-Dependent Dehydratases Using Nonreactive Substrate Mimics. Proc. Natl. Acad. Sci. 2019, 116 (35), 17245. https://doi.org/10.1073/pnas.1905240116.

(18) Ortega, M. A.; Hao, Y.; Zhang, Q.; Walker, M. C.; van der Donk, W. A.; Nair, S. K. Structure and Mechanism of the TRNA-Dependent Lantibiotic Dehydratase NisB. Nature 2015, 517 (7535), 509-512. https://doi.org/10.1038/nature13888.

(19) Cogan, D. P.; Hudson, G. A.; Zhang, Z.; Pogorelov, T. V.; van der Donk, W. A.; Mitchell, D. A.; Nair, S. K. Structural Insights into Enzymatic [4+2] Aza-Cycloaddition in Thiopeptide Antibiotic Biosynthesis. Proc Natl Acad Sci USA 2017.

(20) Wever, W. J.; Bogart, J. W.; Baccile, J. A.; Chan, A. N.; Schroeder, F. C.; Bowers, A. A. Chemoenzymatic Synthesis of Thiazolyl Peptide Natural Products Featuring an Enzyme-Catalyzed Formal [4 + 2] Cycloaddition. J. Am. Chem. Soc. 2015, 137 (10), 3494-3497. https://doi.org/10.1021/jacs.5b00940.

(21) Chalker, J. M.; Gunnoo, S. B.; Boutureira, O.; Gerstberger, S. C.; Fernández-González, M.; Bernardes, G. J. L.; Griffin, L.; Hailu, H.; Schofield, C. J.; Davis, B. G. Methods for Converting Cysteine to Dehydroalanine on Peptides and Proteins. Chem. Sci. 2011, 2 (9), 1666-1676. https://doi.org/10.1039/C1SC00185J.

(22) Wright, T. H.; Bower, B. J.; Chalker, J. M.; Bernardes, G. J. L.; Wiewiora, R.; Ng, W.-L.; Raj, R.; Faulkner, S.; Vallée, M. R. J.; Phanumartwiwath, A.; et al. Posttranslational Mutagenesis: A Chemical Strategy for Exploring Protein Side-Chain Diversity. Science 2016, aag1465. https://doi.org/10.1126/science.aag1465.

(23) Galan, S. R. G.; Wickens, J. R.; Dadova, J.; Ng, W.-L.; Zhang, X.; Simion, R. A.; Quinlan, R.; Pires, E.; Paton, R. S.; Caddick, S.; et al. Post-Translational Site-Selective Protein Backbone a-Deuteration. Nat. Chem. Biol. 2018, 14 (10), 955-963. https://doi.org/10.1038/s41589-018-0128-y.

(24) Bhushan, A.; Egli, P. J.; Peters, E. E.; Freeman, M. F.; Piel, J. Genome Mining- and Synthetic Biology-Enabled Production of Hypermodified Peptides. Nat. Chem. 2019, 11 (10), 931-939. https://doi.org/10.1038/s41557-019-0323-9.

(25) Moon, K.; Xu, F.; Seyedsayamdost, M. R. Cebulantin, a Cryptic Lanthipeptide Antibiotic Uncovered Using Bioactivity-Coupled HiTES. Angew. Chem. 2019, 131 (18), 6034-6038. https://doi.org/10.1002/ange.201901342. 
(26) Quijano, M. R.; Zach, C.; Miller, F. S.; Lee, A. R.; Imani, A. S.; Künzler, M.; Freeman, M. F. Distinct Autocatalytic a-N-Methylating Precursors Expand the Borosin RiPP Family of Peptide Natural Products. $J$. Am. Chem. Soc. 2019, 141 (24), 9637-9644. https://doi.org/10.1021/jacs.9b03690.

(27) Lee, H.; Choi, M.; Park, J.-U.; Roh, H.; Kim, S. Genome Mining Reveals High Topological Diversity of $\omega$-Ester-Containing Peptides and Divergent Evolution of ATP-Grasp Macrocyclases. J. Am. Chem. Soc. 2020, 142 (6), 3013-3023. https://doi.org/10.1021/jacs.9b12076.

(28) Santos-Aberturas, J.; Chandra, G.; Frattaruolo, L.; Lacret, R.; Pham, T. H.; Vior, N. M.; Eyles, T. H.; Truman, A. W. Uncovering the Unexplored Diversity of Thioamidated Ribosomal Peptides in Actinobacteria Using the RiPPER Genome Mining Tool. Nucleic Acids Res. 2019, 47 (9), 4624-4637. https://doi.org/10.1093/nar/gkz192.

(29) Bushin, L. B.; Clark, K. A.; Pelczer, I.; Seyedsayamdost, M. R. Charting an Unexplored Streptococcal Biosynthetic Landscape Reveals a Unique Peptide Cyclization Motif. J. Am. Chem. Soc. 2018, 140 (50), 17674-17684. https://doi.org/10.1021/jacs.8b10266.

(30) Caruso, A.; Bushin, L. B.; Clark, K. A.; Martinie, R. J.; Seyedsayamdost, M. R. Radical Approach to Enzymatic $\beta$-Thioether Bond Formation. J. Am. Chem. Soc. 2019, 141 (2), 990-997. https://doi.org/10.1021/jacs.8b11060.

(31) Clark, K. A.; Bushin, L. B.; Seyedsayamdost, M. R. Aliphatic Ether Bond Formation Expands the Scope of Radical SAM Enzymes in Natural Product Biosynthesis. J. Am. Chem. Soc. 2019, 141 (27), 10610-10615. https://doi.org/10.1021/jacs.9b05151.

(32) Acedo, J. Z.; Bothwell, I. R.; An, L.; Trouth, A.; Frazier, C.; van der Donk, W. A. OMethyltransferase-Mediated Incorporation of a $\beta$-Amino Acid in Lanthipeptides. J. Am. Chem. Soc. 2019, 141 (42), 16790-16801. https://doi.org/10.1021/jacs.9b07396.

(33) Ting, C. P.; Funk, M. A.; Halaby, S. L.; Zhang, Z.; Gonen, T.; van der Donk, W. A. Use of a Scaffold Peptide in the Biosynthesis of Amino Acid-Derived Natural Products. Science 2019, 365 (6450), 280. https://doi.org/10.1126/science.aau6232.

(34) Zhang, Z.; van der Donk, W. A. Nonribosomal Peptide Extension by a Peptide Amino-Acyl TRNA Ligase. J. Am. Chem. Soc. 2019, 141 (50), 19625-19633. https://doi.org/10.1021/jacs.9b07111.

(35) Caruso, A.; Martinie, R. J.; Bushin, L. B.; Seyedsayamdost, M. R. Macrocyclization via an ArginineTyrosine Crosslink Broadens the Reaction Scope of Radical S-Adenosylmethionine Enzymes. J. Am. Chem. Soc. 2019, 141 (42), 16610-16614. https://doi.org/10.1021/jacs.9b09210.

(36) Morinaka, B. I.; Lakis, E.; Verest, M.; Helf, M. J.; Scalvenzi, T.; Vagstad, A. L.; Sims, J.; Sunagawa, S.; Gugger, M.; Piel, J. Natural Noncanonical Protein Splicing Yields Products with Diverse $\beta$-Amino Acid Residues. Science 2018, 359 (6377), 779. https://doi.org/10.1126/science.aao0157.

(37) Hudson, G. A.; Burkhart, B. J.; DiCaprio, A. J.; Schwalen, C. J.; Kille, B.; Pogorelov, T. V.; Mitchell, D. A. Bioinformatic Mapping of Radical S-Adenosylmethionine-Dependent Ribosomally Synthesized and Post-Translationally Modified Peptides Identifies New $\mathrm{Ca}, \mathrm{C} \beta$, and $\mathrm{C} \gamma$-Linked Thioether-Containing Peptides. J. Am. Chem. Soc. 2019, 141 (20), 8228-8238. https://doi.org/10.1021/jacs.9b01519. 